\title{
Optimal Charging of Li-lon Batteries Based on an Electrolyte Enhanced Single Particle Model
}

\author{
Xianke Lin \\ Department of Automotive, Mechanical and \\ Manufacturing Engineering, \\ University of Ontario Institute of Technology, \\ Oshawa, Canada \\ Xiaoguang Hao \\ Department of Mechanical Engineering, \\ University of Michigan, Ann Arbor, USA
}

\begin{abstract}
Lithium ion batteries play important roles as energy storage solutions in electric vehicle, portable devices, and renewable energy systems. There are many issues facing lithium ion batteries. One of them is the long charging time due to the slow electrochemical dynamics. Fast charging is one of the most difficult techniques that affect the acceptance of the electric vehicles. This paper presents a single particle battery model for charging optimization. The single particle model is enhanced with electrolyte dynamics. An optimal charging problem is formulated based on the electrolyte enhanced single particle model. Safety constraints are identified and imposed on the optimal charging problem. Multiple techniques have been developed to reduce the computational load. The fast charging strategy is developed. The results show that the fast charging strategy includes multiple phases and is able to reduce the charge time significantly.
\end{abstract}

Keywords-fast charging; single particle model; Li-ion battery

\section{INTRODUCTION}

Limited fossil fuel reserve and global warming provide a strong driving force for transportation electrification and clean energy technology. Lithium ion batteries play an important role as the most widely used energy storage system, and therefore are essential to the sustainable development of our society. There are still many issues facing lithium ion batteries. One of them is the long charging time due to the slow electrochemical dynamics. Compared with conventional vehicle which refuels in couple minutes, electric vehicle takes several hours to recharge. Battery recharge also requires meticulous control due to complex electrochemical reactions, immeasurable internal states, and serious safety concerns. It is thus important and also the focus of this article to develop a systematic approach solving for the optimal charging strategy while ensuring the safety during the charging process.

\author{
Zhenyu Liu \\ Department of Mechanical Engineering, \\ Zhejiang University, Hangzhou, China \\ Weiqiang Jia \\ Department of Mechanical Engineering, \\ Zhejiang University, Hangzhou, China
}

There are many charging protocols in the literature, such as constant-current / constant-voltage $(\mathrm{CC} / \mathrm{CV})$, pulse current charging, multi-stage charging [4], constant power charging, pulse voltage charging [1], [2], [3], neural networks[5], and fuzzy logic[6,7]. The most widely used charging protocol is the constant-current / constant-voltage $(\mathrm{CC} / \mathrm{CV})$. These approaches use predefined charging profiles with fixed current, voltage, or power constraints. Therefore, they are simple to implement. Most of them are based on the basic knowledge or experience of battery dynamics. These charging methods don't provide the best achievable performance determined by the electrochemistry.

This paper aims to develop a systematical approach for solving the optimal charging strategy based on a single particle model with electrolyte dynamics. An optimal control problem is mathematically formulated. The fast charging strategy is investigated. Multiple techniques, including time to SOC domain conversion and electrolyte dynamics approximation, have been developed in this paper to reduce the computational burden while maintaining the accuracy.

The rest of the paper is structured as follows. In section II, the physics-based single particle model is presented, and the electrolyte dynamics is simplified and added to the single particle model. The model is validated against experimental data. In section III, the optimal charging problem is formulated, and the fast charging strategy is then derived and investigated. In section IV, the optimized fast charging strategy is discussed in details. Section V concludes the presented work.

\section{EELECTROLyte ENHANCED Single Particle Model}

In the following we describe in detail the single particle model with electrolyte dynamics.

A graphite/LiFePO4 cell with $400 \mathrm{mAh}$ is considered in this study. The single particle approach is used here. In the SP model, electrodes are assumed to consist of spherical intercalation particles with identical size. The surface of the spherical particle is scaled to that of the porous electrode. The key assumption in the single particle model is that the current 
distribution is taken to be uniform along the thickness of the electrode. Figure 1 provides a schematic of the SP model with electrolyte dynamics.

The mass balance of lithium ions in an intercalation particle of electrode active material is described by Fick's second law in a spherical coordinate system. Here the diffusion equation is simplified by using two-term polynomial approximation [8-10] as follows:

Cathode:

$$
\begin{aligned}
& \frac{d x_{p, a v g}}{d t}=\frac{-3 J_{p}}{F R_{p} c_{p, \max }}, J_{p}=\frac{I_{a p p}}{S_{p}} \\
& x_{p, \text { surf }}-x_{p, \text { avg }}=\frac{-J_{p} R_{p}}{5 F D_{s, p} c_{p, \max }}
\end{aligned}
$$

Anode:

$$
\begin{aligned}
& \frac{d x_{n, \text { avg }}}{d t}=\frac{-3 J_{n}}{F R_{n} c_{n, \max }}, J_{n}=\frac{I_{a p p}}{S_{n}} \\
& x_{n, \text { surf }}-x_{n, \text { avg }}=\frac{-J_{n} R_{n}}{5 F D_{s, n} c_{n, \max }}
\end{aligned}
$$

where $x_{i, a v g}$, electrode's SOC, is the ratio of the solid average concentration to the maximum solid concentration $c_{i, \max }$ for each electrode. $\mathrm{J}_{\mathrm{i}}$ is the current density for each electrode. $S_{i}$ is the electroactive surface area for each electrode. $I_{\text {app }}$ is the applied current. $R_{i}$ is the particle radius. $x_{i, s u r f}$ is the ratio of the solid surface concentration to the maximum solid concentration. $D_{s, i}$ is the solid phase diffusion coefficient. $F$ is the Faraday constant.

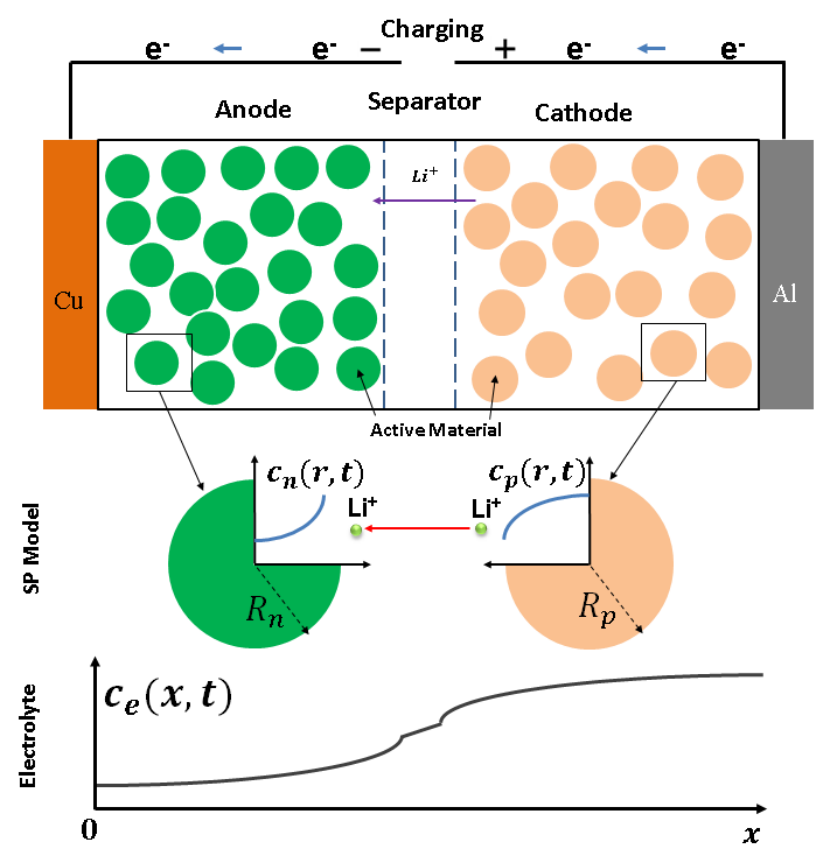

Figure 1. Electrolyte enhanced single particle model

The rate of lithium intercalation and deintercalation reactions is related to the surface SOC and over potential, and is expressed through the Butler-Volmer equation as follows.

$$
J_{i}=k_{i}\left[\exp \left(\frac{\alpha_{a, i} F}{R T} \eta_{i}\right)-\exp \left(\frac{-\alpha_{c, i} F}{R T} \eta_{i}\right)\right]
$$

where $\eta_{i}$ is the over-potential for lithium ion deintercalation/intercalation reactions. $\alpha_{\mathrm{a}, \mathrm{i}}$ and $\alpha_{\mathrm{c}, \mathrm{i}}$ are the anodic transfer coefficient and cathodic transfer coefficient respectively. $\mathrm{R}$ is the gas constant. $\mathrm{T}$ is the temperature. $\mathrm{k}_{\mathrm{i}}$ is the reaction rate coefficient.

$\eta_{\mathrm{p}}$ and $\eta_{\mathrm{n}}$ can be estimated as[11]

$$
\eta_{i}=\frac{R T}{\alpha_{a}} \ln \left(\xi_{i}+\sqrt{\xi_{i}^{2}+1}\right)
$$

where

$$
\xi_{i}=\frac{J_{i}}{2 k_{i}}
$$

The over-potentials are calculated by the following equations

$$
\begin{aligned}
& \eta_{p}=\phi_{\mathrm{p}}-\mathrm{U}_{p}^{\theta} \\
& \eta_{n}=\phi_{n}-\mathrm{U}_{n}^{\theta}
\end{aligned}
$$

where $\phi_{i}$ is the electrode's potential. $U_{i}^{\theta}$ is the open circuit potential in the appendix.

The cell voltage equals to the difference of solid phase potential between the positive and negative electrodes plus the voltage loss due to the internal resistance.

$$
V_{\text {cell }}=\phi_{\mathrm{p}}-\phi_{\mathrm{n}}+I_{\text {app }} R_{\text {cell }}
$$

where $R_{\text {cell }}$ is the internal resistance.

The material balance for the binary electrolyte in the liquid phase is given by [12]

$$
\begin{gathered}
\frac{\partial\left(\varepsilon_{2} c_{e}\right)}{\partial t}=\frac{\partial}{\partial x}\left(D_{e}^{e f f} \frac{\partial}{\partial x} c_{e}\right)+\frac{1-t_{+}^{0}}{F} J_{i} \\
\left.\frac{\partial c_{e}}{\partial x}\right|_{x=0}=\left.\frac{\partial c_{e}}{\partial x}\right|_{x=L}=0
\end{gathered}
$$

where $c_{e}$ is the lithium ion concentration, $\varepsilon_{2}$ is the electrolyte phase volume fraction, $D_{e}^{e f f}$ is the effective electrolyte phase $\mathrm{Li}$ diffusion coefficient. $t_{+}^{0}$ is the transference number.
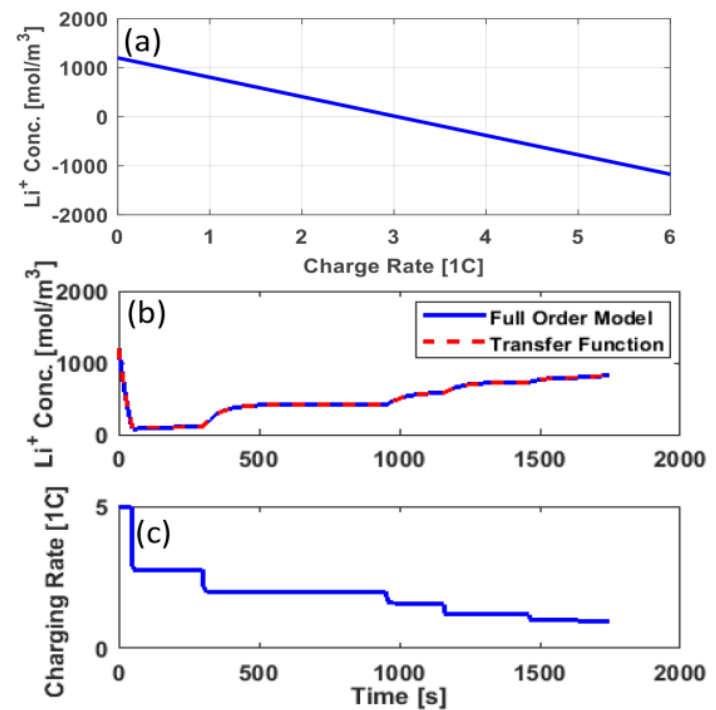

Figure 2. (a) Equilibrium $\mathrm{Li}^{+}$concentration at different charge rates. (b) Comparison of $\mathrm{Li}^{+}$concentration responses between full order model and transfer function. (c) Input current profile to both full order model and transfer function. 
During charging, the longer the diffusion distance of the $\mathrm{Li}^{+}$ ions, the greater the decrease of the $\mathrm{Li}^{+}$concentration in the electrolyte. The lowest electrolyte phase lithium ion concentration occurs near the anode current collector (the diffusion distance is longest from the cathode). Therefore, the lithium depletion could occur near the anode current collector. Based on the full-order model in our previous work [12], a static map is generated to predict the equilibrium $\mathrm{Li}^{+}$ concentration at the anode current collector surface (here the diffusion distance is longest) at different charging rates as shown in Figure 2. The $\mathrm{Li}^{+}$concentration decreases at the anode current collector as the charge rate increases. It reaches 0 $\mathrm{mol} / \mathrm{m}^{3}$ when charge rate reaches about $2.9 \mathrm{C}$ as shown in Figure 2. When the charge current continues to increase beyond $2.9 \mathrm{C}$, the equilibrium $\mathrm{Li}^{+}$concentration decreases to negative values. This is impossible and meaningless in reality. However, it's possible in the simulation, because the concentration is just a continuous variable. Although the negative equilibrium $\mathrm{Li}^{+}$concentration beyond $2.9 \mathrm{C}$ is meaningless in reality, it is actually very useful for predicting concentration decreasing rate at high charge rates. The lower the negative equilibrium lithium concentration at a charging rate, the greater the decreasing rate of the current lithium concentration at that charging rate.

TABLE I. BATTERY MODEL PARAMTERS

\begin{tabular}{|l|c|c|c|}
\hline \multicolumn{1}{|c|}{ Parameter } & $\begin{array}{c}\text { Negative } \\
\text { electrode }\end{array}$ & Separator & $\begin{array}{c}\text { Positive } \\
\text { electrode }\end{array}$ \\
\hline Electrode thickness $(\mathrm{m})$ & $70 \times 10^{-6}$ & $20 \times 10^{-6}$ & $90 \times 10^{-6}$ \\
\hline Particle radius $R_{s}(\mathrm{~m})$ & $3 \times 10^{-6}$ & & $0.2 \times 10^{-6}$ \\
\hline Electroactive surface area $S_{i}\left(\mathrm{~m}^{2}\right)$ & 0.8458 & & 12.6641 \\
\hline Active material volume fraction $\varepsilon_{1}$ & 0.5384 & & 0.4179 \\
\hline Filler volume fraction $\varepsilon_{3}$ & 0.172 & & 0.172 \\
\hline electrolyte phase volume fraction $\varepsilon_{2}$ & 0.2896 & 1 & 0.4101 \\
\hline Max. concentration $c_{i, m a x}\left(\mathrm{~mol} \mathrm{~m}^{-3}\right)$ & 26390 & & 22806 \\
\hline Initial electrode's SOC, $x_{i, a v g}$ & 0.017 & & 0.7862 \\
\hline Li diffusion coefficient $D_{s, i}\left(\mathrm{~m}^{2} \mathrm{~s}^{-1}\right)$ & $3.9 \times 10^{-14}$ & & $2 \times 10^{-17 \mathrm{ad}}$ \\
\hline Initial electrolyte concentration $\left(\mathrm{mol} \mathrm{m}^{-3}\right)$ & 1200 & 1200 & 1200 \\
\hline Charge transfers coefficient $\alpha_{a}, \alpha_{c}$ & 0.5 & & 0.5 \\
\hline Reaction rate coefficient $k_{i}\left(\mathrm{Am}^{-2}\right)$ & $9.77 \times 10^{-2}$ & & $1.19 \times 10^{-2}$ \\
\hline $\begin{array}{l}\text { Time constant for diffusion dynamics } \Delta T \\
\text { (s) }\end{array}$ & 60 & 60 & 60 \\
\hline
\end{tabular}

ad: adjusted

The nonlinear electrolyte diffusion equation has high fidelity. However, it's very computationally costly due to its PDE nature. Therefore, a first-order transfer function is used to approximate the Li concentration dynamics at anode current collector. The first-transfer function is given as follows.

$$
G(s)=\frac{c_{e}(s)}{c_{e_{-} e q}(s)}=\frac{1}{\Delta T s+1}
$$

where $c_{e}(s)$ is the instantaneous lithium concentration (in Laplace domain) at anode current collector. $c_{e_{-} e q}(s)$ is the equilibrium lithium concentration at a given charge rate. $\Delta \mathrm{T}$ is the time constant for the diffusion dynamics.

The transfer function in Laplace domain can be converted into time domain as follows

$$
\frac{d c_{e}}{d t}=\frac{1}{\Delta T}\left(c_{e_{-} e q}-c_{e}\right)
$$

The above first-order equation is able to accurately predict the lithium concentration evolution at the anode current collector. In this equation, lower negative $\mathrm{c}_{\mathrm{e}_{-} \text {eq }}$ will lead to lower negative value of $\frac{\mathrm{dc}_{\mathrm{e}}}{\mathrm{dt}}$, which means greater lithium ion decreasing rate. Therefore, a negative value beyond $2.9 \mathrm{C}$ in Figure 2 indicates a high decreasing rate of $\mathrm{c}_{\mathrm{e}}$. A comparison between transfer function prediction and the full-order model[12] prediction is shown in Figure 2. A charging current profile is input into both transfer function and full-order model. And the resultant $\mathrm{Li}^{+}$concentration profiles at anode current collector are compared. As shown in Figure 2, the transfer function has a very high accuracy (almost 100\%) of predicting the $\mathrm{Li}^{+}$concentration compared with full-order model. Therefore the diffusion dynamics of electrolyte can be simplified by using a static map of equilibrium lithium concentration at different charge rates and a first-order transfer function. This technique developed in this paper significantly reduces the computational cost, and also the states needed in the optimal charging problem.

The battery model parameters are summarized in Table I and more details can be found in [12-14].

The single particle model with electrolyte dynamics is validated against experiment data. The battery cells used are graphite $/ \mathrm{LiFePO}_{4}$ cells with $400 \mathrm{mAh}$ nominal capacity. As shown in Figure 3, a current profile is applied to both the single particle model and the real battery cell. Voltage responses from both simulation and experiment are compared. The RMSE of voltage is about $41 \mathrm{mV}$.

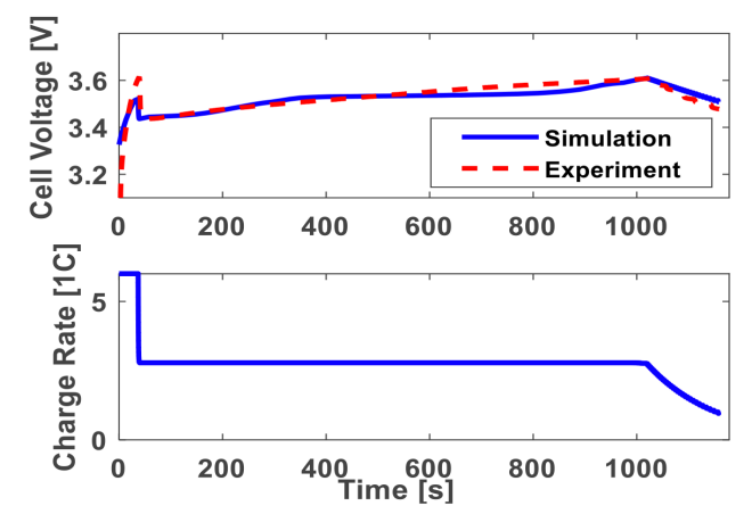

Figure 3. Experiment results for fast charging strategy.

\section{CHARGING STRATEGY OPTIMIZATION}

Based on the validated single particle model, an optimal charging problem is formulated with the aim to reduce the charge time while ensuring the safety constraints. The cost function is given by 


$$
\min _{I(t), t_{f}} \int_{t_{0}}^{t_{f}} 1 \cdot d t
$$

where $\left(t_{f}-t_{0}\right)$ is the charge time. The optimization variables are the input current $I(t)$ and final time $t_{f}$, with the state variables, $x_{i, \text { avg }}$ (electrode's SOC) and $c_{2}$ (electrolyte concentration).

The above optimal charging problem is formulated over time domain. The final time is a variable. To further reduce the state variables and optimization variables, the problem is converted from time domain into SOC domain. In this paper, the anode electrode SOC, $\mathrm{x}_{\mathrm{n}, \mathrm{avg}}$, is used to represent the SOC for the battery cell. When the battery is being charged, the anode SOC starts at a very low level (close to 0 ), and increases to a target high level. Therefore, we can formulate the cost function over anode SOC domain instead of time domain. Anode SOC is first divided in to many small segments $\triangle$ SOC. Over each $\triangle$ SOC segment, the charging time can be calculated by the following equation.

$$
t_{\text {char }}=\frac{\Delta S O C \cdot S_{n} \cdot F \cdot R_{n} \cdot c_{n, \max }}{3 \cdot I(S O C)}
$$

where $t_{\text {char }}$ is the charge time for the segment $\Delta S O C$. Therefore, the new cost function in anode SOC domain becomes

$$
\min _{I(S O C)} \int_{S O C_{l o}}^{S O c_{h i}} t_{c h a r}
$$

where anode electrode $\mathrm{SOC}_{\mathrm{lo}}=0.017$ (fully discharged) and $\mathrm{SOC}_{h i}=0.61$ (fully charged). After the time to SOC conversion, the state variable SOC is eliminated, and optimization variable $t_{f}$ is also eliminated. The optimization variable is now the input current I(SOC) only. The only state variable is the electrolyte concentration $\mathrm{C}_{\mathrm{e}}$ at anode current collector. The above time to SOC conversion technique simplifies the optimal control problem, and reduces the states and control inputs.

The optimal control problem has the following constraints for model dynamics, inputs, and states.

$$
\begin{gathered}
0<\mathrm{I}(\mathrm{t}) \leq \mathrm{I}_{\text {max }} \\
\quad c_{e}>0 \\
0 \leq x_{n, \text { surf }} \leq 1 \\
0 \leq x_{p, \text { surf }} \leq 1 \\
V_{\text {cell }}<4 V
\end{gathered}
$$

These constrains protect the active particle from overcharge (saturation on anode and depletion on cathode), electrolyte from depletion and cell voltage from overvoltage.

Dynamic Programming (DP) is a very powerful, general tool for solving sequential decision making problems. DP as an extremely powerful approach has been extensively applied to real-world optimal control problems, including hybrid vehicle control system[15], path planning for autonomous vehicles[16], hydroelectric generation scheduling[17], etc...

\section{OPTIMIZATION RESULTS}

In this section, the optimal charging problem is solved by dynamic programming. The optimized charging strategy is studied and discussed in details.

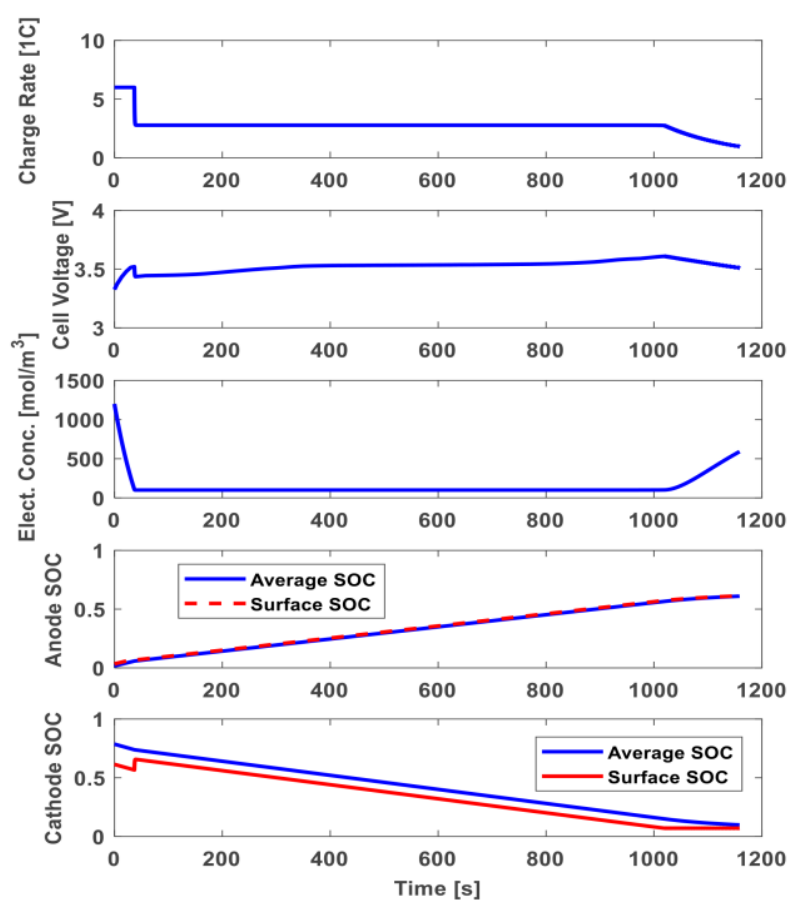

Figure 4. Optimization results for fast charging strategy.

The charging strategy pursued by this work aims to charge a battery as fast as possible. Dynamic programming is applied to find the optimal charging strategy which achieves the minimum charging time. The optimized charging current trajectory is shown in Figure 4. It takes about 19 minutes 18 seconds to achieve a target anode SOC of $61 \%$ (fully charged) from an initial anode SOC $1.7 \%$ (fully discharged).

As shown in Figure 4, the optimized charging process can be divided into three stages. In the first stage, a high maximum current is applied until the $\mathrm{Li}^{+}$concentration in the electrolyte at the anode current collector depletes. In the second stage, the current is lowered to a level to avoid lithium depletion in the electrolyte and maintain a constant and small $\mathrm{Li}^{+}$concentration in the electrolyte at the anode current collector. In the third stage (close to the end of charge), the lithium concentration on the cathode surface decreases and gets close to zero, the charge current is gradually lowered to avoid the lithium depletion on the cathode surface. The whole charging process follows constant-current / constant-electrolyte-concentration / constantcathode-concentration (CC/CEC/CCC).

\section{CONCLUSION}

This paper enhances the single particle model with electrolyte dynamics. Based on the enhanced model, the fast charging strategy is investigated. An optimal charging problem is formulated to solve for the fast charging strategy that achieves the minimum charging time. Dynamic programming as an extremely powerful approach has been used to find the optimal fast charging strategy. Multiple techniques, including electrolyte dynamics approximation and time to SOC domain 
conversion, have been used in this paper to reduce the computational cost and dimensions of the problem for the dynamic programming. Fast charging strategy is investigated and discussed in details. This charging strategy takes the shortest time to charge the battery, about 19 minutes 18 seconds in this study. The fast charging strategy can be divided into three stages and follows constant-current / constantelectrolyte-concentration / constant-cathode-concentration (CC/CEC/CCC).

\section{ACKNOWLEDGMENT}

This works was supported by the NSERC Discovery Program.

\section{APPENDIX}

The equilibrium potentials are obtained by $\mathrm{C} / 50$ charge and discharge, as shown in Figure A1.
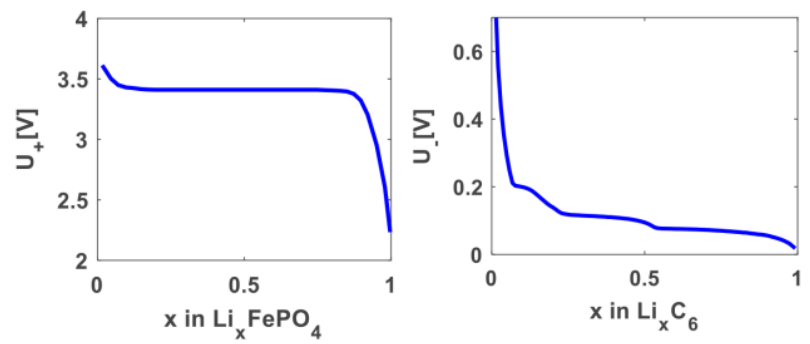

Figure A1. Equilibrium potentials for anode and cathode

\section{REFERENCES}

[1] Notten, P.H., J.O. het Veld, and J. Van Beek, Boostcharging Li-ion batteries: A challenging new charging concept. Journal of Power Sources, 2005. 145(1): p. 89-94.

[2] Purushothaman, B. and U. Landau, Rapid charging of lithium-ion batteries using pulsed currents a theoretical analysis. Journal of The Electrochemical Society, 2006. 153(3): p. A533-A542.

[3] Smith, K.A., Electrochemical control of lithium-ion batteries [applications of control]. IEEE Control Systems, 2010. 30(2): p. $18-25$.

[4] Anseán, D., et al., Fast charging technique for high power lithium iron phosphate batteries: A cycle life analysis. Journal of Power Sources, 2013. 239: p. 9-15.
[5] Ullah, Z., B. Burford, and S. Dillip, Fast intelligent battery charging: neural-fuzzy approach. IEEE Aerospace and Electronic Systems Magazine, 1996. 11(6): p. 26-34.

[6] Surmann, H., Genetic optimization of a fuzzy system for charging batteries. IEEE Transactions on Industrial Electronics, 1996. 43(5): p. 541-548.

[7] Liu, Y.-H. and Y.-F. Luo, Search for an optimal rapid-charging pattern for Li-ion batteries using the Taguchi approach. IEEE Transactions on Industrial Electronics, 2010. 57(12): p. 3963-3971.

[8] Rahimian, S.K., S. Rayman, and R.E. White, State of charge and loss of active material estimation of a lithium ion cell under low earth orbit condition using Kalman filtering approaches. Journal of the Electrochemical Society, 2012. 159(6): p. A860-A872.

[9] Wang, C., W. Gu, and B. Liaw, Micro - Macroscopic Coupled Modeling of Batteries and Fuel Cells I. Model Development. Journal of the Electrochemical Society, 1998. 145(10): p. 34073417.

[10] Ramadass, P., et al., Development of first principles capacity fade model for Li-ion cells. Journal of the Electrochemical Society, 2004. 151(2): p. A196-A203.

[11] Di Domenico, D., G. Fiengo, and A. Stefanopoulou. Lithium-ion battery state of charge estimation with a Kalman filter based on a electrochemical model. in Control Applications, 2008. CCA 2008. IEEE International Conference on. 2008. Ieee.

[12] Lin, X., et al., A comprehensive capacity fade model and analysis for Li-ion batteries. Journal of The Electrochemical Society, 2013. 160(10): p. A1701-A1710.

[13] Arora, P., M. Doyle, and R.E. White, Mathematical Modeling of the Lithium Deposition Overcharge Reaction in Lithium - Ion Batteries Using Carbon - Based Negative Electrodes. Journal of The Electrochemical Society, 1999. 146(10): p. 3543-3553.

[14] Safari, M., et al., Multimodal physics-based aging model for life prediction of Li-ion batteries. Journal of The Electrochemical Society, 2009. 156(3): p. A145-A153.

[15] Lin, C.-C., et al., Power management strategy for a parallel hybrid electric truck. IEEE transactions on control systems technology, 2003. 11(6): p. 839-849.

[16] Flint, M., M. Polycarpou, and E. Fernandez-Gaucherand, Cooperative path-planning for autonomous vehicles using dynamic programming. IFAC Proceedings Volumes, 2002. 35(1): p. 481486.

[17] Chang, S.-C., et al., Hydroelectric generation scheduling with an effective differential dynamic programming algorithm. IEEE transactions on power systems, 1990. 5(3): p. 737-743. 